\title{
ESTUDIO FARMACOEPIDEMIOLÓGICO DE USO DE ANTIINFLAMATORIOS NO ESTEROIDEOS EN PACIENTES DE ALTO RIESGO CARDIOVASCULAR
}

\author{
Jorge Enrique Machado-Alba ${ }^{1,2, a}$, Verónica Alzate-Carvajal'1,2,b, Luis Echeverri-Cataño1,2,c
}

RESUMEN

Con el objetivo de determinar la frecuencia de uso prolongado de antiinflamatorios no esteroideos (AINES) en pacientes colombianos de alto riesgo cardiovascular (ARC) se desarrolló un estudio retrospectivo en el cual se identificaron pacientes de ARC que usaron AINES por más de cinco meses continuos entre enero de 2011 y marzo de 2013. Se identificó a los pacientes que recibían crónicamente nitratos, digitálicos, y clopidogrel y ácido acetil salicílico (ASA), quienes fueron identificados como de ARC. Se realizó un análisis de frecuencias de uso según la comedicación recibida. Se encontró uso concomitante de AINES en el $0,35 \%$ de los consumidores de nitratos (tiempo promedio: 9,5 $\pm 4,4$ meses), en el $0,36 \%$ de los consumidores de clopidogrel y ASA (tiempo promedio: 9,3 $\pm 3,4$ meses), y en el 0,4\% de los consumidores de digitálicos (10,2 $\pm 4,6$ meses) Se concluye que existe una baja proporción de uso de AINES de manera crónica en pacientes de alto riesgo cardiovascular.

Palabras clave: Enfermedades cardiovasculares; Antiinflamatorios no esteroideos; Farmacoepidemiología; Farmacovigilancia; Colombia (fuente: DeCS BIREME).

\section{PHARMACOEPIDEMIOLOGICAL STUDY OF THE USE OF NON-STEROID ANTIINFLAMATORY DRUGS IN HIGH-RISK CARDIOVASCULAR PATIENTS}

\begin{abstract}
In order to determine the frequency of extended use of non-steroidal anti-inflammatory drugs (NSAID) in Colombian patients with high cardiovascular risk (HCVR), a retrospective study was developed which identified HCVR patients who used NSAID for over five continuous months from January 2011 and March 2013. Patients chronically receiving nitrates, digitalis and clopidogrel and acetylsalicylic acid (ASA), known as HCVR, were identified. An analysis of the frequencies of use based on the co-medication received was performed. Concomitant use of NSAID was found in $0,35 \%$ of consumers of nitrates (average time: $9,5 \pm 4,4$ months), in $0,36 \%$ of consumers of clopidogrel and ASA (average time: $9,3 \pm 3,4$ months), and in $0,4 \%$ of consumers of digitalis $(10,2 \pm 4,6$ months). It is concluded that there is a low proportion of chronic use of NSAID in high-risk cardiovascular patients.
\end{abstract}

Key words: Cardiovascular diseases; Anti-Inflammatory agents, non-steroidal; Pharmacoepidemiology; Pharmacovigilance; Colombia (source: MeSH NLM).

\section{INTRODUCCIÓN}

Al formular una prescripción médica se debe tomar en cuenta las características clínicas del paciente, las comorbilidades y la comedicación que se pueda estar generando, para determinar los riesgos más importantes a los que se pueda estar sometido al paciente ${ }^{(1,2)}$. Este riesgo se incrementa cuando no se cuenta con prescripción médica alguna. Varía, evidentemente, con cada fármaco empleado. Se ha estimado que cerca del $70 \%$ de los pacientes mayores de 65 años usan con frecuencia algún antiinflamatorio no esteroideos (AINES), bajo prescripción médica o sin ella ${ }^{(3,4)}$. El uso de AINES ha sido ampliamente discutido por sus efectos adversos tanto cardiovasculares como gastrointestinales ${ }^{(3)}$.

Los AINES se han asociado a reacciones adversas importantes, siendo las más frecuentes las de tipo gastrointestinal, aunque es cada vez más común encontrar complicaciones cardiovasculares como eventos tromboembólicos, infarto del miocardio y eventos

\footnotetext{
Grupo de investigación en Farmacoepidemiología y Fármacovigilancia, Universidad Tecnológica de Pereira. Pereira, Colombia.

Audifarma S.A. Pereira, Colombia.

Médico, máster en Farmacoepidemiología; ${ }^{b}$ estudiante de Medicina; ${ }^{c}$ médico

Recibido: 06-08-2013 Aprobado: 18-09-13
}

Citar como: Machado-Alba JE, Alzate-Carvajal V, Echeverri-Cataño L. Estudio farmacoepidemiológico de uso de antiinflamatorios no esteroideos en pacientes de alto riesgo cardiovascular. Rev Peru Med Exp Salud Publica. 2013;30(4):626-9. 
cerebrovasculares (ECV) ${ }^{(3,5,6)}$. El uso crónico (mayor de 180 días) de inhibidores selectivos de la ciclooxigenasa 2 (COX-2) o de AINES clásicos con selectividad sobre esta isoenzima (diclofenaco), en pacientes con antecedentes de enfermedad cardiovascular y ECV, se ha asociado a mayor riesgo de presentar eventos cardiovasculares, por lo que en este tipo de pacientes se debe tener mayores precauciones para su uso ${ }^{(7-9)}$. Se ha descrito, por ejemplo, que el rofecoxib puede duplicar el riesgo de presentación de eventos trombóticos, comparados con un placebo, cuando se usa por más de 18 meses. Asimismo, se ha descrito que después de su uso continuo por más de 5 meses existía un aumento de más de cuatro veces en la frecuencia de presentar edema pulmonar o falla cardiaca congestiva. Del mismo modo, se ha observado que el uso de AINES, por un periodo de 5 meses o más, incrementa el riesgo de precipitar falla cardíaca se incrementa en pacientes con enfermedad cardíaca isquémica ${ }^{(10)}$.

En Colombia se han conducido estudios que han señalado el uso prolongado -en algunos casos por periodos superiores a 18 meses- de inhibidores selectivos de la COX-2 en pacientes con algún factor de riesgo cardiovascular (11). Sin embargo, no se han descrito la frecuencia de uso concomitante de AINES en pacientes con alto riesgo cardiovascular (ARC). EI objetivo de este estudio fue determinar la frecuencia de uso prolongado de AINES en pacientes afiliados al Sistema General de Seguridad Social en Salud (SGSSS) de Colombia con alto riesgo cardiovascular.

\section{EL ESTUDIO}

Se llevó a cabo un estudio retrospectivo. La información se obtuvo de la base de datos de Audifarma S.A, empresa que dispensa medicamentos a 6,2 millones de personas, que corresponde al $14,1 \%$ de la población colombiana y al $32,6 \%$ de los pacientes afiliados al Sistema General de Seguridad Social en Salud (SGSSS). En colaboración con el departamento de fármacoepidemiología del SGSSS, se recolectaron los datos de todas las prescripciones dadas entre enero de 2011 y marzo de 2013, las cuales fueron discriminadas por cliente institucional, usuario y medicamento. Para ello, se empleó la herramienta Business Object (SAP AG, Alemania) sobre una plataforma en Oracle (Oracle Corporation, EE. UU.).

Se calificó como paciente de ARC a aquel paciente que recibía nitratos, digitálicos o la siguiente combinación de antiagregantes plaquetarios: clopidogrel y ácido acetil salicílico (ASA) de manera crónica, ya que estos medicamentos son indicativos de enfermedad cardíaca isquémica ${ }^{(10)}$. Se incluyó a todos los pacientes de ARC que recibieron, de manera concomitante a la medicación señalada, AINES por cinco meses o más. Para la identificación de los medicamentos involucrados en el estudio se usó la denominación común internacional de estos. El análisis estadístico incluyo el cálculo de las frecuencias absolutas y relativas, y la determinación de las medidas de tendencia central para las variables de interés. Para ello se empleó el programa Microsoft Excel $201{ }^{\circledR}$.

El estudio contó con la aprobación del Comité de Bioética de la Universidad Tecnológica de Pereira, en la categoría de investigación sin riesgo. Para guardar el anonimato de los pacientes incluidos en el estudio, durante la recolección de los datos no se recogió datos que permitieran su identificación.

\section{HALLAZGOS}

Se identificaron un total de 33417 pacientes que recibían algunos de los medicamentos indicativos de enfermedad cardiaca isquémica, el 29,8\% (9969) de ellos eran usuarios de digitálicos; el 52,7\% (17 607), de antiagregantes plaquetarios combinados; y el 17,5\%, de digitálicos.

Entre los usuarios de nitratos, se encontró que el 0,4\% (35/9969) recibió de manera concomitante algún AINES por más de cinco meses de manera continuada. En ellos, el tiempo promedio de uso concomitante fue de $9,5 \pm 4,4$ meses (rango: 5-21), la media de edad fue de $64,9 \pm 14,8$ años (rango: $33-91$ ) y fueron de sexo femenino en su mayoría (54,3\%). En este grupo se observó, además, que entre los pacientes que usaron concomitantemente AINES, el 22,9\% (8/35) recibió simultáneamente dos AINES por al menos un mes. Los pacientes restantes recibieron uno o más AINES, que pudieron ser cambiados en diferentes meses, pero de manera individual. El AINES más usado en este grupo fue el naproxeno, seguido del ibuprofeno y diclofenaco $(77,1 ; 42,8$ y $40 \%$, respectivamente). Cinco pacientes recibieron solamente naproxeno en combinación con los nitratos (media: 12,6 meses), mientras que 22 lo hicieron en promedio por 6,9 \pm 4,8 meses, pero alternados con otros medicamentos del mismo grupo. Adicionalmente, dos pacientes recibieron algún inhibidor selectivo de la COX-2.

En el grupo de usuarios de antiagregantes plaquetarios combinados (clopidogrel + ASA), se encontró un uso concomitante con algún AINES en el 0,4\% (64/17607). En ellos, el tiempo promedio de uso concomitante fue de 9,3 $\pm 3,4$ meses (rango: 5-21), la media de edad fue de $67,2 \pm 14,2$ años (rango: 18-91) y fueron de sexo femenino en su mayoría (60,9\%). Además, en este grupo se observó que el 23,4\% (15/64) recibió una combinación de dos AINES entre uno y cinco meses de forma concomitante. Al igual que en el grupo anterior, los pacientes restantes recibieron uno o más AINES, que pudieron ser cambiados en diferentes meses, pero de manera individual. En este grupo, el AINES usado con más frecuencia también fue el naproxeno, seguido de ibuprofeno y diclofenaco $(78,1 ; 34,3$ y $34,3 \%$ respectivamente). Catorce pacientes recibieron solamente naproxeno en combinación con los antiagregantes (media: 8,4 meses), mientras que los restantes 36 lo hicieron en promedio por 6,8 \pm 7,0 meses, 
pero alternados con otros medicamentos del mismo grupo. Además, tres pacientes recibieron algún inhibidor selectivo de la COX-2.

En el caso del grupo de digitálicos, se encontró un uso concomitante con algún AINES en el 0,4\% (22/5841). En ellos, el tiempo promedio de uso concomitante fue de 10,2 \pm 4,6 meses (rango: 5-21), la media de edad fue de 75,0 \pm 12,8 años (rango: 48-96) y fueron de sexo masculino en su mayoría $(52,2 \%)$. Además, en este grupo se observó que el 9,1\% (2/22) recibió una combinación de dos AINES entre uno y cinco meses, de forma concomitante. Al igual que en el grupo anterior, los pacientes restantes recibieron uno o más AINES, que pudieron ser cambiados en diferentes meses, pero de manera individual. En este grupo, el AINES usado con más frecuencia también fue el naproxeno, seguido de ibuprofeno y diclofenaco $(78,3 ; 34,8$ y $21,7 \%$ respectivamente). Ocho pacientes recibieron solamente naproxeno en combinación con los AINES (media: 10,0 meses), mientras que los restantes diez lo hicieron en promedio por 8,1 $\pm 5,7$ meses, pero alternados con otros medicamentos del mismo grupo. Además, un paciente recibió algún inhibidor selectivo de la COX-2.

\section{DISCUSIÓN}

Se ha descrito que el riesgo de presentar un evento coronario agudo y la mortalidad se incrementa con el uso de AINES en pacientes con riesgo cardiovascular o infarto previo ${ }^{(7)}$. Del total de pacientes que tenían prescripciones para AINES y concomitantemente nitratos, digitálicos o antiagregantes, se encontró la asociación en el $0,4 \%$ de esa población, una proporción de pacientes pequeña comparada con el universo tomado, lo cual sugiere la facilidad de realización de una intervención para disminuir el riesgo en estos pacientes.

En Colombia se realizó un estudio para determinar los riesgos de posibles efectos negativos asociados a la medicación entre usuarios de inhibidores selectivos de la COX-2 con riesgo cardiovascular, donde se encontró que 81 pacientes habían recibido celecoxib y etoricoxib por un tiempo promedio de 8,1 meses. En nuestro estudio, se encontró que solo seis pacientes recibían inhibidores selectivos de la COX-2 más nitratos, antiagregantes o digitálicos. Lo cual puede significar que se está valorando mejor el mencionado riesgo y este grupo de AINES se está empleando menos entre pacientes con enfermedades cardiovasculares ${ }^{(11)}$.

En este estudio se encontró que el naproxeno es el AINES prescrito con mayor frecuencia en los pacientes con ARC. Este, en especial, presenta dos a tres veces menos riesgo cardiovascular que rofecoxib o celecoxib y otros AINES (por ejemplo diclofenaco) y es el preferido en aquellos pacientes que requieren un antiinflamatorio por largos periodos que, además, presenta problemas cardiacos, cerebrovasculares o factores de riesgo para eventos tromboembólicos; sin embargo, no reduce el riesgo de reacciones adversas gastrointestinales (12-15). Aunque la evidencia aún es poco concluyente para determinar la seguridad de los AINES, continúa siendo el naproxeno el medicamento de este grupo con menor riesgo cardiovascular ${ }^{(14)}$. El ibuprofeno fue el segundo AINES con mayor frecuencia de prescripción en pacientes con $A R C$, el cual se asocia de manera similar a otros fármacos del grupo con reacciones adversas cardiovasculares y presenta, adicionalmente, una interacción farmacológica con el ASA, que disminuye su efecto cardioprotector (16). Por otro lado, el diclofenaco, por su alta selectividad a la COX-2 y sus efectos dosis dependientes a nivel del corazón y vasos sanguíneos, se ha descrito como el AINES tradicional con mayor riesgo cardiovascular y cerebrovascular, e incluso algunos estudios señalan que incrementa el riesgo de muerte tan solo con 7 a 14 días de uso en pacientes con un infarto de miocardio previo ${ }^{(8,10,11)}$; las dosis altas de diclofenaco, e incluso de ibuprofeno, se comparan con el riesgo que representan los inhibidores de la COX-2 ${ }^{(16)}$. Ello se debería a que el diclofenaco produciría la inhibición de los canales $L$ de calcio y de los canales de sodio en los células cardiacas produciendo alteraciones en su relajación y contractilidad, pudiendo -en corazones no sanos- desencadenar falla cardiaca ${ }^{(17,18)}$.

Pese a que la proporción de uso de AINES en pacientes con ARC fue baja, se sigue presentando tal situación que eleva la probabilidad de que los pacientes con alguna enfermedad cardíaca puedan presentar un nuevo evento tromboembólico o precipitar una falla cardíaca. Sin embargo, es necesario enfatizar que la mayoría de pacientes con ARC en este estudio, estuvieran recibiendo naproxeno, el medicamento con la menor asociación de eventos trombóticos y el AINES más recomendado para esta condición.

Este trabajo tiene algunas limitaciones que incluyen que la muestra proviene de una población afiliada al SGSSS; por lo tanto, nuestro estudio solo permite sacar conclusiones respecto a grupos con características epidemiológicas similares. Los resultados se obtuvieron a partir de una base de datos poblacional de dispensación de medicamentos que no recoge características clínicas y de severidad de la enfermedad cardiovascular de los pacientes. Es evidente que existe el riesgo de complicaciones cardio y cerebrovasculares en pacientes que reciben AINES, pero es importante resaltar que la frecuencia de esta interacción en una muestra significativa de pacientes colombianos fue bastante baja, y fue notable que en los pacientes con riesgo se empleara naproxeno con mayor frecuencia dado su menor efecto sobre el sistema cardiovascular, lo cual puede significar que se ha incorporado este conocimiento entre los clínicos del país. Sin embargo, es preocupante que aún haya pacientes con cardiopatía isquémica que reciban diclofenaco, o incluso, ibuprofeno. 
Se concluye que existe una baja proporción de uso de AINES de manera crónica en pacientes de alto riesgo cardiovascular. Cabe recomendar la importancia de que se implementen medidas continuas de identificación de los pacientes con riesgo cardiovascular para definir claramente la comedicación que pueden recibir y prevenir oportunamente la aparición de eventos adversos cardiacos.

Contribuciones de autoría: JEM contribuyó con la concepción y diseño del artículo, la obtención de resultados y del análisis e interpretación de datos. VAC contribuyó con el análisis e interpretación de datos y la obtención de resultados. LEC contribuyó con contribuyó con la concepción y diseño del artículo y del análisis e interpretación de datos. Todos los autores participaron de la redacción del artículo y de la aprobación de la versión final.

Fuentes de financiamiento: Universidad Tecnológica de Pereira y Audifarma S.A.

Declaración de conflictos de interés: los autores expresan que no existe ningún conflicto de interés.

\section{REFERENCIAS BIBLIOGRÁFICAS}

1. Fiorucci S, Santucci L, Distrutti E. NSAIDs, coxibs, CINOD and H2Sreleasing NSAIDs: what lies beyond the horizon. Dig Liver Dis. 2007;39(12):104351.

2. Paulose-Ram R, Hirsch R, Dillon C, $\mathrm{Gu} \mathrm{Q}$. Frequent monthly use of selected non-prescription and prescription non-narcotic analgesics among U.S. adults. Pharmacoepidemiol Drug Saf. 2005;14(4):257-66.

3. Ritter JM, Harding I, Warren JB. Precaution, cyclooxygenase inhibition, and cardiovascular risk. Trends Pharmacol Sci. 2009;30(10):503-8.

4. Olsen AM, Fosbol EL, Lindhardsen J, Andersson C, Folke F, Nielsen MB, et al. Cause-specific cardiovascular risk associated with nonsteroidal antiinflammatory drugs among myocardial infarction patients--a nationwide study. PloS one. 2013;8(1):e54309.

5. Lamberts M, Fosbol EL, Olsen AM, Hansen ML, Folke F, Kristensen SL, et al. Ongoing treatment with nonsteroidal anti-inflammatory drugs at time of admission is associated with poorer prognosis in patients with first-time acute myocardial infarction. Int $\mathrm{J}$ Cardiol. Int J Cardiol. 2013;168(2):832-7. doi: 10.1016/j.ijcard.2012.10.004.

6. Huang WF, Hsiao FY, Wen YW, Tsai YW. Cardiovascular events associated with the use of four nonselective NSAIDs (etodolac, nabumetone, ibuprofen, or naproxen) versus a cyclooxygenase-2 inhibitor (celecoxib): a population-based analysis in Taiwanese adults. Clin Ther. 2006;28(11):1827-36.
7. Farkouh ME, Greenberg BP. An evidencebased review of the cardiovascular risks of nonsteroidal anti-inflammatory drugs. Am J Cardiol. 2009;103(9):1227-37.

8. Schjerning Olsen AM, Fosbol EL, Lindhardsen J, Folke F, Charlot M, Selmer $\mathrm{C}$, et al. Duration of treatment with nonsteroidal anti-inflammatory drugs and impact on risk of death and recurrent myocardial infarction in patients with prior myocardial infarction: a nationwide cohort study. Circulation. 2011;123(20):222635 .

9. Bresalier RS, Sandler RS, Quan H, Bolognese JA, Oxenius B, Horgan K, et al. Cardiovascular events associated with rofecoxib in a colorectal adenoma chemoprevention trial. N Engl J Med. 2005;352(11):1092-102.

10. Machado-Alba JE Giraldo C, Ruiz AF. Farmacovigilancia de riesgo cardiovascular por antiinflamatorios no esteroideos COX-2 selectivos. Investigaciones Andina. 2012;14(24):427-36.

11. McGettigan P, Henry D. Use of nonsteroidal anti-inflammatory drugs that elevate cardiovascular risk: an examination of sales and essential medicines lists in low-, middle-, and high-income countries. PLoS Med. 2013;10(2):e1001388. doi: 10.1371/journal.pmed.1001388.

12. Ong HT, Ong LM, Tan TE, Chean KY. Cardiovascular effects of common analgesics. Med J Malaysia. 2013;68(2):189-94.

13. Trelle $S$, Reichenbach S, Wandel S, Hildebrand $\mathrm{P}$, Tschannen B, Villiger PM, et al. Cardiovascular safety of non-steroidal antiinflammatory drugs: network meta-analy- sis. BMJ. 2011;342:c7086. doi: 10.1136/ bmj.c7086.

14. McGettigan P, Henry D. Cardiovascular risk with non-steroidal anti-inflammatory drugs: systematic review of populationbased controlled observational studies. PLoS Med. 2011;8(9):e1001098.

15. Coxib and traditional NSAID Trialists' (CNT) Collaboration, Bhala N, Emberson J, Merhi A, Abramson S, Arber N, et al. Vascular and upper gastrointestinal effects of non-steroidal anti-inflammatory drugs: meta-analyses of individual participant data from randomised trials. Lancet. 2013;382(9894):769-79. doi: 10.1016/ S0140-6736(13)60900-9.

16. Yarishkin OV, Hwang EM, Kim D, Yoo JC, Kang SS, Kim DR, et al. Diclofenac, a Non-steroidal Anti-inflammatory Drug, Inhibits L-type Ca Channels in Neonatal Rat Ventricular Cardiomyocytes. Korean J Physiol Pharmacol. 2009;13(6):437-42. doi: 10.4196/kjpp.2009.13.6.437.

17. Kristóf A, Husti Z, Koncz I, Kohajda Z, Szél T, Juhász V, et al. Diclofenac prolongs repolarization in ventricular muscle with impaired repolarization reserve. PLoS One. 2012;7(12):e53255. doi: 10.1371/ journal.pone.0053255.

Correspondencia: Jorge Enrique Machado Alba Dirección: Calle $105 N^{\circ}$ 14-140, Pereira, Risaralda, Colombia

Teléfono: $57+3108326970$,

Correoelectrónico:machado@utp.edu.co 